\title{
Manifestation of extrinsic spin Hall effect in superconducting structures: Nondissipative magnetoelectric effects
}

\author{
F. Sebastian Bergeret ${ }^{1,2}$ and Ilya V. Tokatly $\mathrm{y}^{3,4}$ \\ ${ }^{1}$ Centro de Física de Materiales (CFM-MPC), Centro Mixto CSIC-UPV/EHU, Manuel de Lardizabal 4, E-20018 San Sebastián, Spain \\ ${ }^{2}$ Donostia International Physics Center (DIPC), Manuel de Lardizabal 5, E-20018 San Sebastián, Spain \\ ${ }^{3}$ Nano-Bio Spectroscopy group, Dpto. Física de Materiales, Universidad del País Vasco, Av. Tolosa 72, E-20018 San Sebastián, Spain \\ ${ }^{4}$ IKERBASQUE, Basque Foundation for Science, E-48011 Bilbao, Spain \\ (Received 16 May 2016; revised manuscript received 17 October 2016; published 7 November 2016)
}

\begin{abstract}
We present a comprehensive quasiclassical approach for studying transport properties of superconducting diffusive hybrid structures in the presence of extrinsic spin-orbit coupling. We derive a generalized Usadel equation and boundary conditions that in the normal state reduce to the drift-diffusion theory governing the spin-Hall effect in inversion symmetric materials. These equations predict the nondissipative spin-galvanic effect, that is the generation of supercurrents by a spin-splitting field, and its inverse - the creation of magnetic moment by a supercurrent. These effects can be seen as counterparts of the spin-Hall, anomalous Hall, and their inverse effects in the superconducting state. Our theory opens numerous possibilities for using superconducting structures in magnetoelectronics.
\end{abstract}

DOI: 10.1103/PhysRevB.94.180502

The spin-orbit coupling (SOC) in normal systems is at the basis of striking magnetoelectric effects, such as the spin (SHE) [1] and anomalous (AHE) [2] Hall effects widely studied in normal systems [3]. What the counterparts of these effects are in the superconducting state is, in several aspects, still an open question.

According to its origin the SOC can be classified as intrinsic or extrinsic. Intrinsic SOC generates from the crystal potential associated with the electronic band structure, and in superconducting structures, in analogy with the normal state, might lead to nondissipative magnetoelectric and spingalvanic effects as shown in theoretical studies [4-8]. In contrast, extrinsic SOC originates from a random potential due to impurities. Its influence on the thermodynamics of bulk superconductors was studied long ago by Abrikosov and Gorkov (AG) [9], who explained nonvanishing magnetic susceptibility of superconductors at zero temperature. The AG model has been used later to describe the physics of superconductor-ferromagnet (S-F) structures with SOC. Within this model, the SOC acts only as a relaxation term for the spin in the normal and for triplet correlations in the superconducting state. The suppression of triplet correlations in S-F-S junctions is associated with the suppression of oscillatory behavior of the critical Josephson current $[10,11]$.

It is well established in the theory of normal systems that SOC not only leads to spin relaxation but also to the coupling between spin and charge currents, responsible for extrinsic SHE and AHE. One expects that this coupling translates to a singlet-triplet coupling in the superconducting state, by analogy to the case of noncentrosymmetric superconductors with intrinsic SOC [12]. However, for superconductors with extrinsic SOC this coupling has never been considered, and there is no theoretical framework for its description.

In this Rapid Communication we address this issue and derive from a microscopic model a diffusion equation for superconducting structures with extrinsic SOC. This equation, Eq. (5), generalizes the well known Usadel equation and contains not only the usual relaxation term due to the SOC, but also a coupling between spin and charge degrees of freedom that lead to the SHE and AHE in the normal case. By using the derived equations we demonstrate that the charge-spin coupling indeed translates in the superconducting state into singlet-triplet coupling. Moreover, our equations also show that the lack of a macroscopic inversion symmetry due, for example, to the presence of hybrid interfaces, leads to magnetoelectric effects. An example of these is a magnetic moment induced by a supercurrent. Inversely, SOC leads to the creation of a supercurrent when the system is polarized via the exchange field $h$ of a ferromagnet. In the latter case the magnitude of the induced supercurrent is, as the anomalous Hall voltage, proportional to $h \theta$, where $\theta$ is the $\mathrm{SH}$ angle.

Basic equations for diffusive superconductors with extrinsic $S O C$. We first explain how to derive the generalized Usadel equation and boundary conditions that allow for an accurate description of superconducting diffusive structures with extrinsic SOC [13]. Following the standard derivation of the quasiclassical equations (see, e.g., Ref. [14]) the starting point is the kinetic equation for the Wigner transformed Keldysh $8 \times 8$ matrix Green's function $\check{G}\left(\mathbf{p}, \mathbf{r} ; t, t^{\prime}\right)$,

$$
\tau_{3} \partial_{t} \check{G}+\partial_{t^{\prime}} \check{G} \tau_{3}+\frac{p_{k}}{m} \partial_{k} \check{G}+i\left[\mathbf{h} \sigma \tau_{3}+\check{\Delta}, \check{G}\right]=\mathcal{I},
$$

where $\mathbf{h}$ is the spin-splitting field, $\check{\Delta}$ is the anomalous selfenergy (SE) describing superconducting correlations, and $\tau_{j}$ and $\sigma^{a}$ are Pauli matrices spanning the Nambu and spin spaces, respectively. The collision integral $\mathcal{I}$ in Eq. (1) describes scattering at impurities,

$$
\mathcal{I}=-i[\check{\Sigma}, \check{G}]+\frac{1}{2}\left\{\nabla_{\mathbf{r}} \check{\Sigma}, \nabla_{\mathbf{p}} \check{G}\right\}-\frac{1}{2}\left\{\nabla_{\mathbf{p}} \check{\Sigma}, \nabla_{\mathbf{r}} \check{G}\right\},
$$

where we performed the standard gradient expansion. We describe impurities by an operator $\hat{W}(\mathbf{r})=V(\mathbf{r})+$ $\hat{V}_{s o}(\mathbf{r})$, with $V(\mathbf{r})$ being a random scalar potential, $\hat{V}_{s o}=$ $-i \lambda^{2}(\nabla V(\mathbf{r}) \times \nabla) \sigma$ the SOC term, and $\lambda$ the effective Compton wavelength. Within the Born approximation the SE $\check{\Sigma}(\mathbf{p}, \mathbf{r})$ in Eq. (2) is the Wigner transform of $\check{\Sigma}\left(\mathbf{r}_{1}, \mathbf{r}_{2}\right)=$ $\left\langle\hat{W}\left(\mathbf{r}_{1}\right) \breve{G}\left(\mathbf{r}_{1}, \mathbf{r}_{2}\right) \hat{W}\left(\mathbf{r}_{2}\right)\right\rangle$, where the angular brackets denote averaging over impurities configuration. In $\check{\Sigma}$ we identify two types of terms: (i) those quadratic in the potentials, $\langle V G V\rangle$ 
and $\left\langle\hat{V}_{s o} G \hat{V}_{\text {so }}\right\rangle$, which lead to the relaxation of momentum and spin, respectively, and (ii) the mixed terms $\left\langle V G \hat{V}_{s o}\right\rangle$ that account for the charge-spin coupling. The last terms are traditionally disregarded in the quasiclassical kinetic theory of superconductors $[10,15,16]$. The importance of mixed terms has been recognized in the context of spin transport in normal conductors $[17,18]$, where they are responsible for the extrinsic SHE and the spin current "swapping." Our goal is to incorporate these magnetoelectric effects into the quasiclassical theory of diffusive superconductors, which requires reconsideration of the standard derivation procedure of the quasiclassical equations.

To consistently catch the charge-spin coupling one needs to include gradient terms in the collision integral Eq. (2). This brings momentum derivatives of the GF which do not allow for a straightforward integration over the particle energy $\xi_{p}$ to derive the Eilenberger equation for the quasiclassical GF $\check{g}(\mathbf{n})=\frac{i}{\pi} \int d \xi_{p} \check{G}$ that depends on the direction $\mathbf{n}=\mathbf{p} / p_{F}$ of the Fermi momentum. In order to overcome this difficulty we first obtain from Eq. (1) equations for the zeroth $\sum_{\mathbf{p}} \breve{G}$ and first $\sum_{\mathbf{p}} \mathbf{p} \breve{G}$ moments of the GF. At this level one can introduce the quasiclassical GF and consider directly the diffusive limit in which $\breve{g}(\mathbf{n})$ is approximated as $\breve{g}(\mathbf{n}) \rightarrow \breve{g}+n_{k} \check{g}_{k}$, where $\check{g}$ is the isotropic part and $\check{g}_{k} \ll \check{g}$ is the leading anisotropic correction. The anisotropic part $\check{g}_{k}$ determines the "matrix current"

$$
\check{J}_{k}=v_{F} \check{g}_{k}-\frac{\lambda^{2} p_{F}}{4 \tau} \epsilon_{k j a}\left\{\sigma^{a},\left[\check{g}, \check{g}_{k}\right]\right\},
$$

where the second term is the "anomalous velocity" contribution due to SOC, and $\tau$ is the momentum scattering time. The physical charge and spin currents are obtained from the Keldysh component of the matrix current, $j_{k}=$ $e \pi N_{0} \operatorname{Tr} \tau_{3}{\breve{J_{k}^{K}}}^{K}(t, t) / 4$ and $j_{k}^{a}=\pi N_{0} \operatorname{Tr} \sigma^{a} \breve{J}_{k}^{K}(t, t) / 4$, respectively. In the diffusive limit one can solve the equation for the first moment, and one finds the anisotropic component $\check{g}_{k}$ that allows us to express the matrix current in terms of the isotropic part $\check{g}$ of GF

$$
\check{J}_{k}=-D\left(\check{g} \partial_{k} \check{g}-\frac{\theta}{2} \epsilon_{k j a}\left\{\sigma^{a}, \partial_{j} \check{g}\right\}+i \frac{\kappa}{2} \epsilon_{k j a}\left[\sigma^{a}, \check{g} \partial_{j} \check{g}\right]\right) .
$$

Here $D$ is the diffusion coefficient. In addition to the usual diffusion current, Eq. (4) contains the expected SH angle $\theta=$ $2 \lambda^{2} p_{F} / v_{F} \tau$ and the swapping term $\kappa=2 \lambda p_{F}^{2} / 3$ first described in Ref. [19]. From the equation for zeroth moment of the full GF we find that the isotropic component of the GF, subjected to the normalization condition $\breve{g}^{2}=1$, satisfies the generalized Usadel equation,

$$
\begin{aligned}
& \tau_{3} \partial_{t} \check{g}+\partial_{t^{\prime}} \check{g} \tau_{3}+\partial_{k} \check{J}_{k}+i\left[\mathbf{h} \boldsymbol{\sigma} \tau_{3}+\check{\Delta}, \check{g}\right] \\
& =-\frac{1}{8 \tau_{s o}}\left[\sigma^{a} \check{g} \sigma^{a}, \check{g}\right]+\frac{1}{4} D \theta \epsilon_{k j a}\left[\sigma^{a}, \check{g} \partial_{k} \check{g} \partial_{j} \check{g}\right],
\end{aligned}
$$

where $1 / \tau_{\text {so }}=8 \lambda^{4} p_{F}^{4} / 9 \tau$. Finally, the Kupriyanov-Lukichev boundary conditions [20] at the interface between a conventional BCS superconductor and a metal with extrinsic SOC can be easily generalized by using the matrix current of Eq. (4),

$$
v_{k} \check{J}_{k}=\frac{D}{2 R_{b} \sigma_{0}}\left[\check{g}_{\mathrm{BCS}}, \check{g}\right],
$$

where $v$ is a unit vector normal to the interface, $R_{b}$ is the barrier resistance per area, $\sigma_{0}$ the conductivity of the normal region, and $\check{g}_{\text {BCS }}$ is the bulk superconductor GF.

Equations (4)-(6) are the main results of this paper. They describe the proximity effect in materials with extrinsic SOC. Despite that the derivation relies on Born approximation, where only the side-jump contribution to the SH angle appears $[17,18]$, the final set of Eqs. (4)-(6) is expected to be quite general with $\theta$ and $\kappa$ being the material parameters accounting for all extrinsic and intrinsic (in cubic materials) contributions to the charge-spin coupling. In fact, Eq. (4) can be viewed as a symmetry based gradient expansion of the current.

In the normal state the terms proportional to $\kappa$ and $\theta$ vanish from Eq. (5). These nonlinear in $\breve{g}$ terms do appear only if superconducting correlations are present and may lead to interesting unexplored phenomena.

Nondissipative magnetoelectric effects. We now discuss physical effects predicted by Eqs. (5) and (6). For clarity we assume a weak superconducting proximity effect and linearize the Usadel equation. Moreover, we focus here on nondissipative physics and switch to the equilibrium Matsubara formalism by replacing in Eq. (5) $\partial_{t} \rightarrow \omega=\pi T(2 n+1)$, the Matsubara frequency. After linearization $\breve{g}=\operatorname{sgn}(\omega) \tau_{3}+$ $i \tau_{2} \hat{f}$ the Usadel equation in nonsuperconducting regions reads

$$
D \nabla^{2} \hat{f}-\{[|\omega|+i \mathbf{h} \boldsymbol{\sigma} \operatorname{sgn}(\omega)], \hat{f}\}=\frac{3 \hat{f}-\sigma^{a} \hat{f} \sigma^{a}}{4 \tau_{s o}},
$$

where $\hat{f}=f_{s}+\operatorname{sgn}(\omega) \sigma^{b} f_{t}^{b}$ is the anomalous GF which describes the induced superconducting condensate and consists of the singlet $f_{s}$ and odd-frequency triplet $f_{t}^{b}$ components. The linearized boundary condition (6) now reads

$$
\begin{gathered}
v_{k}\left(\partial_{k} f_{s}-\theta \epsilon_{k j a} \partial_{j} f_{t}^{a}\right)=i \gamma f_{\mathrm{BCS}} \\
v_{k}\left(\partial_{k} f_{t}^{a}-\theta \epsilon_{k j a} \partial_{j} f_{s}-\kappa\left[\partial_{a} f_{t}^{k}-\delta_{k a} \partial_{j} f_{t}^{j}\right]\right)=0,
\end{gathered}
$$

where $\gamma=1 / R_{b} \sigma_{0}$ and $f_{\mathrm{BCS}}=\Delta / \sqrt{\omega^{2}+\Delta^{2}}$. As we can see from Eqs. (7)-(9) the effect of SOC is twofold. On the one hand, the extrinsic SOC leads to the known additional relaxation of the condensate (via the Elliot-Yaffet mechanism), described by the right-hand side of Eq. (7), if the triplet component is nonvanishing. On the other hand, the SOC induces, out of the singlet, the triplet component at the hybrid interfaces, even in the absence of the exchange field h. The term in Eqs. (8) and (9) proportional to the SH angle describes the singlet-triplet conversion, which is the analog to the charge-spin conversion in normal metals. This conversion can be understood as a consequence of inversion asymmetry at the hybrid interface. Due to the antisymmetric tensor $\epsilon_{j k a}$ in the SH term the singlet-triplet conversion occurs only in setups with currents flowing parallel to the interfaces, as for example lateral Josephson junctions that will be discussed below.

As a first example we consider a superconducting film with extrinsic SOC in the absence of the exchange field, $\mathbf{h}=0$. The film occupies the region $-d / 2<z<d / 2$ and is infinite in the $(x, y)$ plane. The region $z>|d / 2|$ is occupied by vacuum 
and hence the boundary condition at $z= \pm d / 2$ is obtained by assuming zero current, i.e., the r.h.s of Eq. (6) vanishes. We assume a small gradient of the superconducting phase $\nabla \varphi=$ $q \hat{\boldsymbol{x}}$ along $x$, so that the singlet component of the anomalous GF is given by $f_{s}(x) \approx i f_{\mathrm{BCS}} e^{i \varphi(x)}$. The triplet component can be easily obtained from Eq. (7) and Eq. (9) which for the present geometry read $\left.\partial_{z} f_{t}^{y}\right|_{ \pm d / 2}=\theta \partial_{x} f_{s} \approx-\theta q f_{\mathrm{BCS}}$. Despite that the film is nonmagnetic $(\mathbf{h}=0)$, the $y$ component of the triplet is generated due to a finite $\mathrm{SH}$ angle $\theta$, and this leads to a finite magnetic moment $m^{y}=\mu_{B} 2 \pi N_{0} T \sum_{\omega} \operatorname{Im}\left[f_{s}^{*} f_{t}^{y}\right]$ :

$$
m^{y}(z)=\mu_{B} \theta T \sum_{\omega} \frac{j_{x}(\omega)}{D k} \frac{\sinh k z}{\cosh k d / 2},
$$

where $j_{x}(\omega)=q \pi D N_{0} f_{\mathrm{BCS}}^{2}$ is the spectral supercurrent, and $k^{2}=k_{\omega}^{2}+k_{s o}^{2}$ with $k_{\omega}^{2}=2|\omega| / D$ and $k_{s o}=1 / D \tau_{s o}$. The induced magnetization Eq. (10) is opposite at opposite sides of the film so that the net magnetic moment is zero, which is a clear consequence of the inversion symmetry. The supercurrent-induced accumulation of the odd-frequency triplet component and the spin density at the film edges is the nondissipative analog of extrinsic SHE.

Let us now consider a normal metal layer $(\mathrm{N})$ of thickness $d$ and finite $\mathrm{SH}$ angle $\theta$, in contact with a bulk superconductor. The $\mathrm{N}$ and $\mathrm{S}$ layers occupy the region $0<z<d$ and $z<0$, respectively. We assume a supercurrent flowing within the $S$ layer due to a small phase gradient $\nabla \varphi=q \hat{\boldsymbol{x}}$. Because of the proximity effect the singlet component penetrates $\mathrm{N}$ where it is converted to a triplet component due to the $\mathrm{SH}$ term in the boundary conditions. Both singlet and triplet components can be easily determined from Eq. (7) and the boundary conditions at the $\mathrm{S} / \mathrm{N}$ interface Eqs. (8) and (9). The induced magnetic moment is then given by:

$$
\begin{aligned}
m^{y}(z)= & \mu_{B} \gamma^{2} \theta T \sum_{\omega} \frac{j_{x}(\omega) \cosh k_{\omega}(z-d)}{D k_{\omega}^{2} k \sinh ^{2} k_{\omega} d \sinh k d} \\
& \times\left[\cosh k z-\cosh k_{\omega} d \cosh k(z-d)\right] .
\end{aligned}
$$

Thus the supercurrent flowing in the $\mathrm{S}$ layer induces a spin density over the whole $\mathrm{N}$ layer. In contrast to our previous example, now the net magnetization is nonzero. In other words, the supercurrent generates a global spin, which is allowed due to the structure inversion asymmetry of the $\mathrm{S} / \mathrm{N}$ bilayer. Phenomenologically this can be described as a nondissipative Edelstein effect (EE). The important difference with the usual EE [4-6] is that it originates solely from the extrinsic SOC and the macroscopic asymmetry of the structure.

Experimentally it might be easier to detect the inverse of this effect, namely, the generation of supercurrents by a combination of SOC and exchange field, which is our third example. We consider a multiterminal lateral $\mathrm{S} / \mathrm{F}$ structure (Fig. 1) which resembles lateral structures used in experiments on SFS structures [21-24] The $n$th $\mathrm{S}$ terminal is infinite in the $y$ direction and has a width $W_{n}$, while $\mathrm{F}$ is a ferromagnet with an exchange field $\mathbf{h}=h \hat{\boldsymbol{y}}$ along $y$. The current density flowing through the $n$th $\mathrm{S} / \mathrm{N}$ interface is readily obtained from Eq. (6):

$$
j_{z}^{(n)}(x)=\frac{\pi T}{e R_{b n}} \sum_{\omega} \operatorname{Im}\left[f_{0}^{(n)}(x) f_{s}^{*}(x, 0)\right]
$$

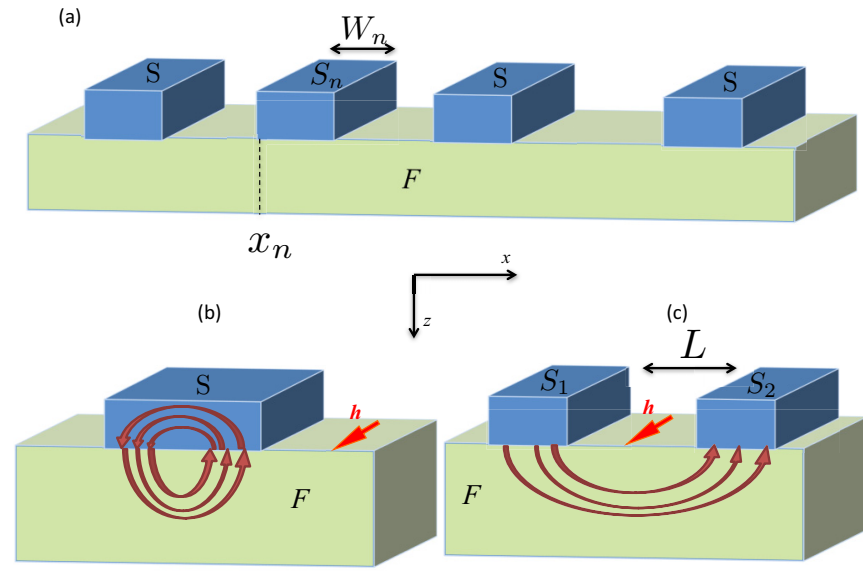

FIG. 1. Lateral S/F structures and illustration of the supercurrent flow.

here $f_{0}^{(n)}(x)=i f_{\mathrm{BCS}} e^{i \varphi_{n}}\left[\Theta\left(x-x_{n}\right)-\Theta\left(x-x_{n}-W_{n}\right)\right]$ is the GF of the $n$th $\mathrm{S}$ electrode with the phase $\varphi_{n}$, and $f_{S}(x)$ is the singlet component induced in $\mathrm{N}$ at $z \rightarrow 0$. If all phases are identical, e.g., $\varphi_{n}=0$ for all $n$, only the real part $f_{s}^{\operatorname{Re}}(x)$ of the singlet GF in $\mathrm{N}$ contributes to the current as in this case $\operatorname{Im}\left[f_{0}^{(n)} f_{s}^{*}\right]=f_{\mathrm{BCS}} f_{s}^{\mathrm{Re}}$. From Eqs. (7)-(9) we find that only for simultaneously nonvanishing $h$ and $\theta$ the component $f_{s}^{\operatorname{Re}}(x)$ can be generated as follows: Due to the proximity effect a purely imaginary "primary" $f_{s}$ is induced in F, where it is converted, via the exchange coupling term $h$ in Eq. (7), into the real triplet $f_{t}^{\mathrm{Re}}$. Finally, $f_{t}^{\mathrm{Re}}$ is converted into $f_{s}^{\mathrm{Re}}$ via the SH term $\theta$ in Eq. (8). Since the SH singlet-triplet coupling involves gradients, it is clear that $f_{s}^{\operatorname{Re}}(x)$ will be generated near inhomogeneities - the edges of the $\mathrm{S}$ terminals. In general the function $f_{s}^{\operatorname{Re}}(x)$ can be written as follows:

$$
f_{s}^{\mathrm{Re}}(x)=k_{h}^{2} \theta \sum_{n=1}^{M} \gamma_{n}\left[s\left(x-x_{n}\right)-s\left(x-x_{n}-W_{n}\right)\right],
$$

where $M$ is the number of terminals, $k_{h}^{2}=2 h / D, \gamma_{n}=$ $1 / R_{b n} \sigma_{0}$, and $s(x)$ is a function localized near the origin and describing the singlet component induced at the left/right edges of each $\mathrm{S}$ electrode. In the limit of thick, formally semi-infinite F layer we find (see Supplemental Material for details)

$$
\begin{aligned}
\frac{s(x)}{f_{\mathrm{BCS}}}= & \frac{k_{-}^{2}-k_{\omega}^{2}}{k_{+}\left(k_{+}^{2}-k_{-}^{2}\right)^{2}} e^{-k_{+}|x|}+\frac{k_{+}^{2}-k_{\omega}^{2}}{k_{-}\left(k_{+}^{2}-k_{-}^{2}\right)^{2}} e^{-k_{-}|x|} \\
& -\frac{2 k_{s o}^{2}}{\pi^{2}\left(k_{+}^{2}-k_{-}^{2}\right)^{2}} \int_{-\infty}^{\infty} d x^{\prime} K_{0}\left(k_{+}\left|x-x^{\prime}\right|\right) K_{0}\left(k_{-}\left|x^{\prime}\right|\right),
\end{aligned}
$$

where $k_{ \pm}^{2}=k_{\omega}^{2}+k_{s o}^{2} / 2 \pm \sqrt{k_{s o}^{4} / 4-k_{h}^{4}}$, and $K_{0}(x)$ is the modified Bessel function of the second kind.

In the one-terminal case $(M=1)$ the right-hand side in Eq. (12) is antisymmetric with respect to the center of the terminal. Therefore the current $j_{z}^{(1)}(x)$ Eq. (11), being also antisymmetric, averages to zero after the integration over $x$. In other words, in a one-terminal $\mathrm{S} / \mathrm{N}$ lateral structure, the combination of the extrinsic SOC and the exchange field generates circulating currents as sketched in Fig. 1(b). 

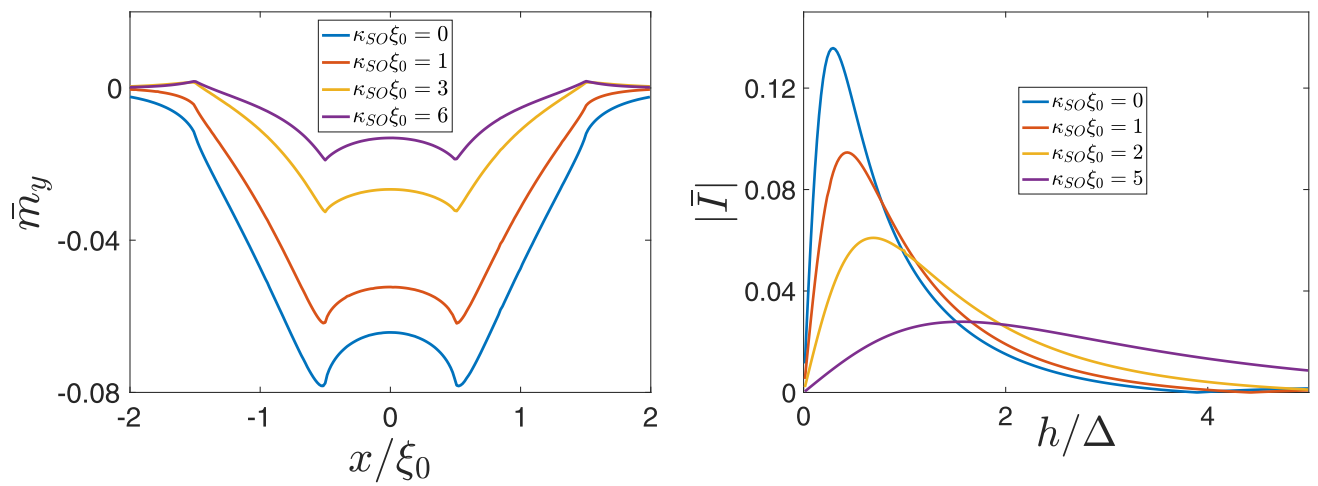

FIG. 2. Left panel: the $x$ dependence of the induced magnetic moment $\bar{m}^{y}=m^{y} /\left(\pi \mu_{B} N_{0} \theta \gamma_{1} \gamma_{2} D\right)$ at $z=0$ for a symmetric lateral SNS junction with $L=1, W=1$, and $\varphi=\frac{\pi}{2}$. Right panel: induced anomalous supercurrent as a function of the exchange field for $L=1$ and $W=5$. We have chosen $T=0.1$ and defined $\xi_{0}=\sqrt{D / 2 \Delta}$.

In the two-terminal case, shown in Fig. 1(c), the total current $I_{1}$ flowing through the $\mathrm{S} 1$ terminal is nonzero due to $f_{s}^{\operatorname{Re}}(x)$ induced from the $\mathrm{S} 2$ terminal:

$$
\begin{aligned}
I_{1}= & \frac{\pi k_{h}^{2} \theta T}{e R_{b 1} R_{b 2} \sigma_{0}} \sum_{\omega} f_{\mathrm{BCS}} \\
& \times \int_{x_{1}}^{x_{1}+W_{1}} d x\left[s\left(x-x_{2}\right)-s\left(x-x_{2}-W_{2}\right)\right] .
\end{aligned}
$$

Therefore besides currents circulating around each interface, there is a finite Josephson current induced by mutual effect of extrinsic SOC and the exchange field [see Fig. 1(c)]. This supercurrent at $\varphi=0$ resembles the anomalous current in a $\varphi_{0}$ junction studied in the context of intrinsic SOC in polar crystals $[5,6,25]$. Here we show that $\varphi_{0}$ junction can be built out of the most common inversion symmetric materials provided they show a finite exchange spin splitting and a $\mathrm{SH}$ angle. The anomalous current is proportional to $\theta h$, which in turn is proportional to the anomalous Hall conductivity $\sigma_{A H}$ in ferromagnets [2]. Hence $\mathrm{F}$ materials with large $\sigma_{A H}$ are good candidates for showing an anomalous supercurrent in lateral SFS structures. If for example one uses a ferromagnet with strong exchange field such that $k_{h}^{2} \gg k_{s o}^{2}$, the amplitude of the anomalous current is according to our theory proportional to $\theta$ times the critical current of the junction. Thus, for materials with $\theta \sim 5-20 \%$ the anomalous phase current can be detected by using quantum interferometer devices as done for example in Ref. [26] for nanowires.

In the right panel of Fig. 2 we show the anomalous current through the S1 terminal as a function of the field $h$. The current starts from zero at $h=0$, reaches a maximum, and finally decays for large fields because of the usual suppression of superconductivity by the $h$ field [11,27]. Inversely, if $h=0$ a finite Josepshon current $(\varphi \neq 0)$ between the two $S$ electrodes induces a finite magnetic moment (see SM for details) similar to the situation found in the $\mathrm{S}$ and $\mathrm{S} / \mathrm{N}$ layered systems. In the left panel of Fig. 2 we show the $x$ dependence of the magnetic moment induced at $z=0$.

In conclusion, we have presented a theoretical framework that describes diffusive superconducting hybrid structures with extrinsic SOC. We have derived equations that contain hitherto unknown terms proportional to the $\mathrm{SH}$ angle, responsible in the normal state for the SHE, and the Lifshits-Dyakonov spincurrents swapping parameter. Our equations explore numerous effects in the field of superconducting spintronics [28-30] and open up numerous opportunities for the control of charge and spin currents in the nondissipative regime. As illustrative examples we demonstrate the existence of magnetoelectric effects in different superconducting structures. We show that these effects are proportional to the $\mathrm{SH}$ angle and hence can be observed by combining materials with known large $\theta$, like Pt or Co, with superconducting electrodes.

The work of F.S.B. was supported by Spanish Ministerio de Economía y Competitividad (MINECO) through the Project No. FIS2014-55987-P and the Basque Government under UPV/EHU Project No. IT-756-13. I.V.T. acknowledges support from the Spanish Grant FIS2013-46159-C3-1-P, and from the "Grupos Consolidados UPV/EHU del Gobierno Vasco" (Grant No. IT578-13)
[1] J. Sinova, S. O. Valenzuela, J. Wunderlich, C. Back, and T. Jungwirth, Rev. Mod. Phys. 87, 1213 (2015).

[2] N. Nagaosa, J. Sinova, S. Onoda, A. MacDonald, and N. Ong, Rev. Mod. Phys. 82, 1539 (2010).

[3] I. Zutic, J. Fabian, and S. Das Sarma, Rev. Mod. Phys. 76, 323 (2004).

[4] V. M. Edelstein, Phys. Rev. Lett. 75, 2004 (1995).

[5] F. S. Bergeret and I. V. Tokatly, Europhys. Lett. 110, 57005 (2015).
[6] F. Konschelle, I. V. Tokatly, and F. S. Bergeret, Phys. Rev. B 92, 125443 (2015).

[7] A. Mal'shukov and C. Chu, Phys. Rev. B 78, 104503 (2008).

[8] A. G. Mal'shukov, S. Sadjina, and A. Brataas, Phys. Rev. B 81, 060502 (2010).

[9] A. A. Abrikosov and L. P. Gorkov, Zh. Exp. Teor. Fiz. 42, 1088 (1962) [Sov. Phys. JETP 15, 752 (1962)].

[10] E. A. Demler, G. B. Arnold, and M. R. Beasley, Phys. Rev. B 55, 15174 (1997). 
[11] F. S. Bergeret, A. F. Volkov, and K. B. Efetov, Rev. Mod. Phys. 77, 1321 (2005).

[12] F. S. Bergeret and I. V. Tokatly, Phys. Rev. B 89, 134517 (2014).

[13] See Supplemental Material at http://link.aps.org/supplemental/ 10.1103/PhysRevB.94.180502 for the detailed derivation of the quasiclassical equations.

[14] D. N. Langenberg and A. I. Larkin, Nonequilibrium Superconductivity (North-Holland, Amsterdam, 1986).

[15] J. Alexander, T. Orlando, D. Rainer, and P. Tedrow, Phys. Rev. B 31, 5811 (1985).

[16] A. F. Volkov, F. S. Bergeret, and K. B. Efetov, Phys. Rev. B 75, 184510 (2007).

[17] R. Raimondi and P. Schwab, Physica E 42, 952 (2010).

[18] K. Shen, R. Raimondi, and G. Vignale, Phys. Rev. B 90, 245302 (2014).

[19] M. B. Lifshits and M. I. Dyakonov, Phys. Rev. Lett. 103, 186601 (2009).

[20] M. Y. Kuprianov and V. F. Lukichev, Pis'ma Zh. Eksp. Teor. Fiz. 94, 139 (1988) [Sov. Phys. JETP 67, 1163 (1988)].
[21] R. S. Keizer, S. T. B. Goennenwein, T. M. Klapwijk, G. Miao, G. Xiao, and A. Gupta, Nature 439, 825 (2006).

[22] M. Anwar, F. Czeschka, M. Hesselberth, M. Porcu and J. Aarts, Phys. Rev. B 82, 100501(R) (2010).

[23] Jian Wang, Meenakshi Singh, Mingliang Tian, Nitesh Kumar, Bangzhi Liu, Chuntai Shi, J. K. Jain, Nitin Samarth, T. E. Mallouk, and M. H. W. Chan, Nat. Phys. 6, 389 (2010).

[24] A. Singh, S. Voltan, K. Lahabi, and J. Aarts, Phys. Rev. X 5, 021019 (2015).

[25] A. I. Buzdin, Phys. Rev. Lett. 101, 107005 (2008).

[26] D. B. Szombati, S. Nadj-Perge, D. Car, S. R. Plissard, E. P. A. M Bakkers, and L. P. Kouwenhoven, Nat. Phys. 12, 568 (2016).

[27] A. I. Buzdin, Rev. Mod. Phys. 77, 935 (2005).

[28] T. Wakamura, N. Hasegawa, K. Ohnishi, Y. Niimi, and Y. Otani, Phys. Rev. Lett. 112, 036602 (2014).

[29] S. Takahashi and S. Maekawa, Jpn. J. Appl. Phys. 51, 010110 (2011).

[30] M. Eschrig, Phys. Today 64(1), 43 (2011); J. Linder, and J. W. A. Robinson, Nat. Phys. 11, 307 (2015). 